\title{
Sphecidae (Hymenoptera: Apoidea) of Rio de Janeiro State (Southeast Brazil): new geographic records and remarks on faunal distribution
}

\author{
Sandor Christiano Buys ${ }^{1,2}$ \\ ${ }^{1}$ Laboratório de Biodiversidade Entomológica, Instituto Oswaldo Cruz, Fundação Oswaldo Cruz, \\ Av. Brasil, 4365, Pavilhão Mourisco, Sala 201, Manguinhos, CEP 21045-900, Rio de Janeiro, RJ, Brazil \\ ${ }^{2}$ Corresponding author: Sandor Christiano Buys, e-mail: sbuys@biologia.ufrj.br, sandor.buys@gmail.com
}

BUYS, S.C. Sphecidae (Hymenoptera: Apoidea) of Rio de Janeiro State (Southeast Brazil): new geographic records and remarks on faunal distribution. Biota Neotrop.11(4): http://www.biotaneotropica.org.br/v11n4/ en/abstract?short-communication+bn02311042011

Abstract: Aiming to improve the database produced during the project Diversity of the Atlantic Forest of the Rio de Janeiro State, new geographic records of sphecid wasps in Rio de Janeiro State are provided. The sphecid fauna found in the sandy coastal plains, lowland forested areas and highland areas on the Serra dos Órgãos are compared and an estimate of the total number of species of Sphecidae from Rio de Janeiro State is provided.

Keywords: solitary wasps, Aculeate, Atlantic Forest, restinga, geographic distribution.

BUYS, S.C. Sphecidae (Hymenoptera: Apoidea) do Estado do Rio de Janeiro (Sudeste do Brasil): novos registros geográficos e notas sobre distribuição da fauna. Biota Neotrop. 11(4): http://www.biotaneotropica. org.br/v11n4/pt/abstract?short-communication+bn02311042011

Resumo: Com o objetivo de incrementar o banco de dados produzido durante o projeto Diversidade da Mata Atlântica do Estado do Rio de Janeiro, novos registros geográficos de vespas esfecídeas no Estado do Rio de Janeiro são fornecidos. A fauna de esfecídeos encontrada em planície costeira arenosa, áreas baixas florestadas e áreas altas da Serra dos Órgãos são comparadas e uma estimativa do número total de espécies encontrada no Estado do Rio de Janeiro é fornecida.

Palavras-chave: vespas solitárias, Aculeata, Mata Atlântica, restinga, distribuição geográfica. 


\section{Introduction}

During the years of 2006 and 2007, the research support foundation of the State of Rio de Janeiro - Fundação Carlos Chagas Filho de Amparo à Pesquisa do Estado do Rio de Janeiro (FAPERJ) - sponsored the project Diversity of the Atlantic Forest of the Rio de Janeiro State, including the inventory of the entomological fauna (Couri et al. 2009). In such occasion, 30 species of Sphecidae (Hymenoptera: Apoidea) were recorded, with base on the review of the pertinent literature and on the examination of the entomological collection of the Museu Nacional - Universidade Federal do Rio de Janeiro (Buys 2009). Moreover, the biological information on the Sphecidae found in Rio de Janeiro State was summarized and several original biological remarks were provided (Buys 2009). Aiming to improve the database on Sphecidae produced during this project, in the present paper new records of geographic occurrence are added, based on the examination of the entomological collection of Instituto Oswaldo Cruz (CEIOC) (Rio de Janeiro, Brazil); besides, the sphecid fauna from sandy coastal plains and forested areas are compared and an estimate of the total number of species in Rio de Janeiro State is provided.

The identification of the species was based especially on the following papers: Willink (1951), Menke (1964, 2004), van der Vecht \& van Breugel (1968), Vardy (1978). Specimens that did not perfectly match with the descriptions in the pertinent literature were not included in the paper.

\section{Results and Discussion}

\section{New records of geographic occurrence of Sphecidae in Rio de Janeiro State}

\section{SUBFAMILY AMMOPHILINAE}

Ammophila gracilis Lepeletier de Saint Fargeau, 1845

Records. Petrópolis, 1, J. Costa, IV.1981 (CEIOC); Rio de Janeiro (Jacarepaguá), 1 q , T.C.M. Gonçalves, I.1982 (CEIOC); Teresópolis (Vargem Grande), 1 우 , M.V. Ferraz, I.1988 (CEIOC).

Eremnophila eximia (Lepeletier de Saint Fargeau, 1845)

Record. Seropédica, 1, L.E.P. Rêgo, 08.III.1983 (CEIOC).

Eremnophila melanaria (Dahlbom, 1843)

Records. Itatiaia (700 m), 19, J.F. Zikán, 4.IV.1926 (CEIOC); idem (900 m), 1ㅇ, 1ð̄, W. Zikán, 2.II.1932 (CEIOC); Teresópolis, 1ㅇ, 1ð̊, J. Jurberg, II.1969 (CEIOC).

\section{SUBFAMILY SPHECINAE}

\section{Prionyx fervens (Linnaeus, 1758)}

Records. Arraial do Cabo, 1 , , J. Jurberg \& P. Jurberg, IV.1963 (CEIOC); Cabo Frio, 2ㅇ, J. Jurberg, IV.1962 (CEIOC); idem, 2今, J. Jurberg, I.1962 (CEIOC).

\section{Prionyx thomae (Fabricius, 1775)}

Records. Cabo Frio, 1q, J. Jurberg, VII.1962 (CEIOC); Rio de Janeiro (Campo Grande), 1 \%, S. Pacheco, III.1969 (CEIOC); Seropédica (Br 101, km 47), 1ठ, 15.XI.1945 (CEIOC); idem, 2§, 29.XI.1945 (CEIOC); idem, 1, 30.IX.1946 (CEIOC); idem, 1, 11.XII.1946 (CEIOC).

Isodontia costipennis (Spinola, 1851)

Records. Itatiaia (700 m), 2ð̂, Zikán, J.F., 15.V.1940 (CEIOC); idem, 1 ㅇ, Zikán, J.F., 08.VI.1941 (CEIOC); idem,
19, J.F. Zikán. 04.VI.1941 (CEIOC); idem, 1Ô, Zikán, J.F. 08.VI.1941 (CEIOC).

Isodontia cyanipennis (Fabricius, 1793)

Records. Itatiaia, 1, J.F. Zikán, 18.V.1940 (CEIOC); idem, 1ð̂, J.F. Zikán, 14.V.1941 (CEIOC); idem, 1ổ, J.F. Zikán, 25.05.1941 (CEIOC).

Sphex caliginosus Erichson, 1849

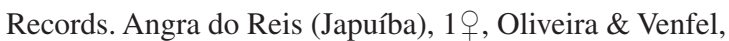
XII.1940 (CEIOC); Itaiaia (700m), 1우, J. F. Zikán (CEIOC); idem, 1, J.F. Zikán, 5.XI.1937 (CEIOC); idem, 1, J. F. Zikán, 24.XI.1946 (CEIOC).

Sphex dorsalis Lepeletier de Saint Fargeau, 1845

Records. Itatiaia (700 m), 19, J.F. Zikán, 16.03.1941 (CEIOC); Rio de Janeiro (Manguinhos), 1운, Lopes, III.1939 (CEIOC); idem, 1ㅇ, 16.I.1915 (CEIOC); Seropédica (Estrada Br 101, km 47), 1오 1ð̊, W. Zikán (CEIOC).

Sphex ichneumoneus (Linnaeus, 1758)

Records. Itatiaia (700 m), 1ð̊, J.F. Zikán, 24.V.1932 (CEIOC); idem, 1ð̂, J.F. Zikán, 18.V.1940 (CEIOC); idem, 2 đ̂, J.F. Zikán, 8.V.1940 (CEIOC); idem, 19, J.F. Zikán, 15.V.1940 (CEIOC); idem, 19, J.F. Zikán, 6.VI.1940 (CEIOC).

Sphex ingens F. Smith, 1856

Records. Angra dos Reis, 1, 33̊; L.T., XII.1932 (CEIOC); Parati, 2 , A. Jesus, II.1974 (CEIOC).

Sphex melanopus Dahlbom, 1843

Records. Angra dos Reis, 1요 II.1916 (CEIOC); Itatiaia (Estação Biológica, 1.800 m), 1§̂, W. Zikán, 27.I.1932 (CEIOC); Teresópolis, 1§̂, J. Jurberg, II.1969 (CEIOC).

Sphex opacus Dahlbom, 1845

Records. Cabo Frio, 1q, J. Junberg, IV.1962 (CEIOC); Itatiaia (700 m), 1 , J.F. Zikán, 29.V.1940 (CEIOC); idem, 1̧̄, J.F. Zikán, 21.V.1941 (CEIOC); Seropédica (Estrada Br 101, Km 47), 1, W. Zikán, 23.I.1947 (CEIOC).

Sphex servillei Lepeletier de Saint Fargeau, 1845

Records. Itatiaia (700 m), 20̂, J.F. Zikán, 15.V.1940 (CEIOC); idem, 19, J.F. Zikán, 06.III.1940 (CEIOC); idem, 1 ㅇ, J.F. Zikán, 19.I.1934 (CEIOC); idem, 1 † , J.F. Zikán, 29.I.1935 (CEIOC).

\section{SUBFAMILY SCELIPHRINAE}

Sceliphron asiaticum (Linnaeus, 1758)

Records. Rio de Janeiro (Manguinhos), 1ㅇ, 18.XI.1913 (CEIOC); idem, 1ㅇ, 02.XI.1914 (CEIOC); idem, 1우, 28.XII.1912 (CEIOC); idem (Collection Adolpho Lutz), 1ㅇ, 30.10.1916 (CEIOC); idem (Collection Adolpho Lutz), 1우 I.1917 (CEIOC); idem, 1옹 A. Jesus, 1963 (CEIOC); idem, 1, J.Jurberg, I.1963 (CEIOC); idem, 1 \%, $1 ð$ (pinned together), J.Jurberg, I.1963 (CEIOC); idem (Collection Adolpho Lutz), 1 ㅇ, 13.I.1910 (CEIOC).

Sceliphron fistularium (Dahlbom, 1843)

Records. Nova Iguaçu, 1 , , T.C. Gonçalves, IV.1979 (CEIOC); Itatiaia (500 m), 3ð̊ J.F. Zikán, 06.XII.1934 (CEIOC); idem, 19, J.F. Zikán, 31.XII.1944 (CEIOC); idem (Coleção J.F. Zikán), 19, I.1945 (CEIOC); idem (Collection J.F. Zikán), 19, 26.XII.1944 (CEIOC); idem 
(Coleção J.F. Zikán), 1ㅇ, 10.II.1933 (CEIOC); Rio de Janeiro (Manguinhos), 1, L.T., II.1932 (CEIOC); Idem, 1ㅇ, J. Jurberg, VIII.1961 (CEIOC); Idem (Collection Adolpho Lutz), 19, X.1911 (CEIOC); Idem (Collection Adolpho Lutz), 1, 13.XI.1920 (CEIOC); idem, 1ㅇ, A. de Jesus, X.1960 (CEIOC); idem, 2q, J. Jurberg, III.1963 (CEIOC); Idem, 1ㅇ, P. Albuquerque \& J. Jurberg, V.1967 (CEIOC); Idem, (Collection Adolpho Lutz, number 3548), 1ð̊, 25.III.1920 (CEIOC); idem (Collection Adolpho Lutz), 1ठ, 12.XII.1912 (CEIOC); idem (Collection Adolpho Lutz), 1 9 , 01.I.1916 (CEIOC); Rio de Janeiro (S. da Piedade), 1, 19.XI.1916 (CEIOC); Rio de Janeiro (Represa dos Ciganos), 1 , , M.V. Ferraz; Rio de Janeiro (Recreio dos Bandeirantes), 1, José Jurberg, 07.X.1960 (CEIOC); Seropédica (Br 101, Km 47), 4ð̊, Zikán, 14.VI.1949 (CEIOC); idem, 19, W. Zikán, X.1949 (CEIOC); Três Rios, 1, Jane M. Costa, III.1980 (CEIOC).

Trigonopsis rufiventris (Fabricius, 1804)

Record. Rio de Janeiro (Grajaú), 1ठ̃, Lopes, 19.VI.1941 (CEIOC).

\section{Comparison among the sphecid fauna of different regions of Rio de Janeiro State}

Although the sandy coastal plains of Rio de Janeiro State are covered by a peculiar vegetational formation known as restinga (Lacerda et al. 1984), the sphecid fauna that inhabit the sandy coastal plains seems to be not peculiar, so that the species recorded in this habitat are also found in adjacent forested areas (Table 1). On the other hand, remarkably the areas of restinga in the Rio de Janeiro State present lower number of species recorded than forested areas (Table 1), even though some areas of restinga had been well sampled, such as the Restinga de Barra de Maricá (City of Maricá). Therefore it seems that the sandy coastal plains from the Rio de Janeiro State are in fact poorer in sphecid species than adjacent forested areas. The genera Penepodium, Trigonopsis and Eremnophila seem do not occur in areas of restinga, or at least they are not conspicuous elements of the fauna in this habitat.

Lowland forested areas are richer in sphecid species recorded than forested areas from Serra dos Órgãos (Table 1), but possibly this is a result of a sample bias, since the lowlands areas apparently are well sampled than the highland places from the Serra dos Órgãos. Species of Isodontia, Penepodium and Podium recorded to Rio de Janeiro State are known only from the Serra dos Órgãos (Table 1), but they are rarely collected species, poorly represented in collections. Prionyx bifoveolatus is the unique species somewhat well collected in Serra dos Órgãos and that has not been found in other places in Rio de Janeiro State (Table 1).

\section{Estimative number of sphecid species in the Rio de Janeiro State}

The examination of the pertinent literature and of the material deposited in collections indicates that at least 47 species of Sphecidae have been found in the Southeast Brazil. Beside the 30 species listed by Buys (2009), the following 17 species of Sphecidae were found in Southeast Brazil, but were not recorded in the Rio de Janeiro State to date: Chlorion hemiprasinum (Sichel, 1863), C. hemipyrrum (Sichel, 1863); C. strandi Willinki, 1951; Eremnophila willinki (Menke, 1964); Penepodium brasiliense (Schrottky, 1903); P. egregium (Saussure, 1867); P. fallax (Kohl, 1902); P. hortivagans (Strand, 1910); P. junonion (Schrottky, 1903); P. paoloense (Schrottky, 1903); Podium denticulatum Smith, 1856; P. eurycephalum Ohl, 1996; P. friesei Kohl, 1902; P. rufipes Fabricius, 1804; Prionyx chilensis (Spinola, 1851); Sphex subhyalinus Fox, 1899;
Table 1. Comparison of the sphecid fauna from three regions of the Rio de Janeiro State with distinct vegetation and geographic aspects, based on geographical records by Buys (2009) and additions from the present paper.

Tabela 1. Comparação entre a fauna de esfecídeos de três regiões do Estado do Rio de Janeiro de vegetação e aspectos geográficos distintos, com base nos registros geográficos de Buys (2009) e adições do presente artigo.

\begin{tabular}{|c|c|c|c|}
\hline & $\begin{array}{l}\text { Coastal } \\
\text { plains } \\
\text { covered } \\
\text { with } \\
\text { restinga } \\
\end{array}$ & $\begin{array}{c}\text { Forested areas } \\
\text { at the sea level } \\
\text { and lowland } \\
\text { places from } \\
\text { Serra do Mar }\end{array}$ & $\begin{array}{l}\text { Serra } \\
\text { dos } \\
\text { Órgãos }\end{array}$ \\
\hline Ammophila gracilis & $\mathrm{X}$ & $\mathrm{X}$ & $\mathrm{X}$ \\
\hline Eremnophila binodis & - & $\mathrm{X}$ & $\mathrm{X}$ \\
\hline Eremnophila eximia & - & $\mathrm{X}$ & $\mathrm{X}$ \\
\hline Eremnophila melanaria & - & $\mathrm{X}$ & $\mathrm{X}$ \\
\hline Eremnophila opulenta & - & $\mathrm{X}$ & - \\
\hline Prionyx bifoveolatus & - & - & $\mathrm{X}$ \\
\hline Prionyx fervens & $\mathrm{X}$ & $\mathrm{X}$ & - \\
\hline Prionyx thomae & $\mathrm{X}$ & $\mathrm{X}$ & $\mathrm{X}$ \\
\hline Isodontia costipennis & - & - & $\mathrm{X}$ \\
\hline Isodontia cyanipennis & - & - & $\mathrm{X}$ \\
\hline Sphex caliginosus & - & $\mathrm{X}$ & $\mathrm{X}$ \\
\hline Sphex dorsalis & $\mathrm{X}$ & $\mathrm{X}$ & $\mathrm{X}$ \\
\hline Sphex ichneumoneus & - & $\mathrm{X}$ & $\mathrm{X}$ \\
\hline Sphex ingens & - & $\mathrm{X}$ & - \\
\hline Sphex latro & - & $\mathrm{X}$ & - \\
\hline Sphex melanopus & - & X & $\mathrm{X}$ \\
\hline Sphex nitidiventris & - & $\mathrm{X}$ & - \\
\hline Sphex opacus & $\mathrm{X}$ & $\mathrm{X}$ & - \\
\hline Sphex servillei & - & $\mathrm{X}$ & $\mathrm{X}$ \\
\hline Dynatus nigripes & - & $X$ & - \\
\hline Penepodium latro & - & $\mathrm{X}$ & - \\
\hline Penepodium luteipenne & - & $\mathrm{X}$ & $X$ \\
\hline Penepodium taschenbergii & - & - & $\mathrm{X}$ \\
\hline Podium sexdentadum & - & - & $\mathrm{X}$ \\
\hline Sceliphron asiaticum & $\mathrm{X}$ & $\mathrm{X}$ & $X$ \\
\hline Sceliphron fistularium & $\mathrm{X}$ & $\mathrm{X}$ & $\mathrm{X}$ \\
\hline Trigonopsis rufiventris & - & $\mathrm{X}$ & - \\
\hline Total number of species & 7 & 22 & 18 \\
\hline
\end{tabular}

Trigonopsis violascens (Dalla Torre, 1897). In addition, it seems that there are a few other species of the genera Isodontia, Penepodium and Podium in Southeast Brazil, but they could not be accurately identified before the conclusion of taxonomic revisionary studies. Therefore, a conservative estimate of the total number of Sphecidae species in the Southeast Brazil could be of about 50-60 species. Since the diversity of environments from the Southeast Brazil Atlantic Forest is well represented in Rio de Janeiro State, the most of this species probably inhabit this State.

\section{Acknowledgements}

I thank Jane Costa (Jane Margaret Costa von Sydow) for facilitating the examination of the entomological collection of the Instituto Oswaldo Cruz and the Fundação Carlos Chagas de Amparo à Pesquisa do Estado do Rio de Janeiro (FAPERJ) for the post-doctoral grant. 
Buys, S.C.

\section{References}

AMARANTE, S.T.P. 2002. A synonymic catalog of the neotropical Crabronidae and Sphecidae (Hymenoptera: Apoidea). Arq. Zool. (São Paulo) 37(1):1-139.

BOHART, R.M. \& MENKE, A.S. 1976. Sphecidae wasps of the world: a generic revision. University of California Press, Berkeley.

BUYS, S.C. 2009. Sphecidae (Hymenoptera: Apoidea) of Rio de Janeiro State (southeast Brazil): inventory of species and notes on biology and distribution. Arq. Mus. Nac. Rio J. 67(3-4):275-282.

COURI, M., NESSIMIAN, J., MEJDALANI, G., MONNÉ, M.L., FRAGA, S.M.L., MENDONÇA, M.C., MONTEIRO, R.F. \& BUYS, S.C. 2009. Levantamento dos insetos da Mata Atlântica do Estado do Rio de Janeiro. Arq. Mus. Nac. Rio J. 67:151-154.

LACERDA, D., ARAúJO, D.S.D., CERQUEIRA, R. \& TURCQ, B., EDS. 1984. Restingas: origem, estrutura e processos. Centro Editorial da Universidade Federal Fluminense, Niterói.
MENKE, A.S. 1964. A new subgenus of Ammophila from the Neotropical Region (Hymenoptera: Sphecidae). Can. Entomol. 96:874-883. http:// dx.doi.org/10.4039/Ent96874-6

MENKE, A.S. 2004. Ammophila hevans Menke, a new species from southern South America, with taxonomic notes on similar species (Hymenoptera: Apoidea: Sphecidae). J. Kansas Entomol. Soc. 77:765-773. http://dx.doi. org/10.2317/E-16.1

van DER VECHT, J. \& van BREUGEL, F.M.A. 1968. Revision of the nominate subgenus Sceliphron Latreille (Hymenoptera, Sphecidae) (Studies on the Sceliphronini, Part I). Tijdschr. Ent. 111:185-255.

VARDY, C.R. 1978. A revision of the Neotropical wasp genus Trigonopsis Perty (Hymenoptera: Sphecidae). Bull. Br. Mus. Ent. 37:117-152.

WILLINK, A. 1951. Las especies argentinas y chilenas de "Chlorionini" (Hym., Sphecidae). Acta. Zool. Lilloana 11:53-225. 